\title{
Emergent Properties of an Organic Semiconductor Driven by its Molecular Chirality
}

Ying Yang, ${ }^{\dagger}$ Beth Rice, ${ }^{\dagger}$ Xingyuan Shi, ${ }^{\dagger}$ Jochen R. Brandt, ${ }^{\ddagger}{ }^{\dagger}$ Rosenildo Correa da Costa, ${ }^{\ddagger}$ Gordon J. Hedley, ${ }^{\S \odot}$ Detlef-M. Smilgies, ${ }^{\|}$Jarvist M. Frost, ${ }^{\perp, \Phi[}$ Ifor. D. W. Samuel, ${ }^{\S}$ Alberto Otero-de-la-Roza, ${ }^{\#}$ Erin R. Johnson, ${ }^{\nabla}$ Kim E. Jelfs, ${ }^{\ddagger}{ }^{\dagger}$ Jenny Nelson, ${ }^{\dagger}$ Alasdair J. Campbell, ${ }^{*}{ }^{\dagger}$ and Matthew J. Fuchter*,*0

${ }^{\dagger}$ Department of Physics and the Centre for Plastic Electronics and ${ }^{*}$ Department of Chemistry and the Centre for Plastic Electronics, Imperial College London, South Kensington Campus, London SW7 2AZ, United Kingdom

- Faculty of Computing, Engineering and Science, University of South Wales, Cemetery Road, Glyntaff, Pontypridd CF37 4BD, United Kingdom

${ }^{\S}$ University of St. Andrews, North Haugh, Fife KY16 9SS, United Kingdom

"Cornell High Energy Synchrotron Source (CHESS), Wilson Laboratory, Cornell University, Ithaca, New York 14853, United States

${ }^{\perp}$ Department of Chemistry, University of Bath, Bath BA2 7AY, United Kingdom

"Department of Chemistry, University of British Columbia, Okanagan, 3247 University Way, Kelowna, British Columbia V1V 1V7, Canada

${ }^{\nabla}$ Department of Chemistry, Dalhousie University, Halifax, Nova Scotia B3H 4R2, Canada

${ }^{\text {II }}$ Department of Materials, Imperial College London, London SW7 2AZ, United Kingdom

\section{Supporting Information}

ABSTRACT: Chiral molecules exist as pairs of nonsuperimposable mirror images; a fundamental symmetry property vastly underexplored in organic electronic devices. Here, we show that organic field-effect transistors (OFETs) made from the helically chiral molecule 1-aza[6] helicene can display up to an 80-fold difference in hole mobility, together with differences in thin-film photophysics and morphology, solely depending on whether a single handedness or a 1:1 mixture of left- and right-handed molecules is employed under analogous fabrication conditions. As the molecular properties of either mirror image isomer are identical, these changes must be a result of the different bulk packing induced by chiral composition.

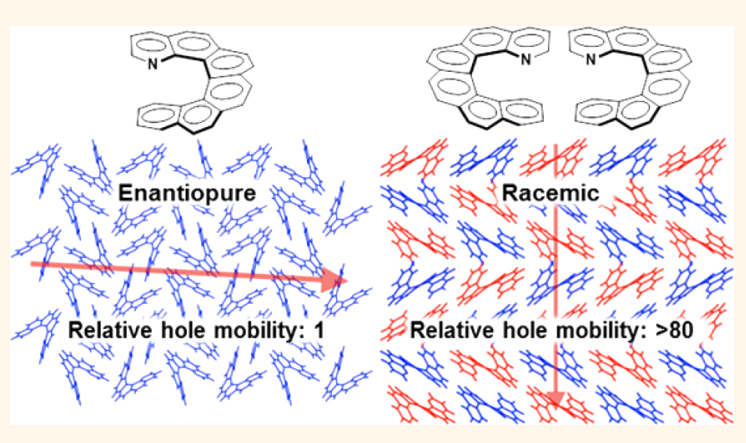
Such underlying structures are investigated using crystal structure prediction, a computational methodology rarely applied to molecular materials, and linked to the difference in charge transport. These results illustrate that chirality may be used as a key tuning parameter in future device applications.

KEYWORDS: chirality, organic semiconductor, helicene, self-assembling, circular polarization, structure prediction

\footnotetext{
$\longrightarrow$ hirality is a fundamental symmetry property, which manifests across multiple length scales, from elemental particles, to molecules and even macroscopic objects, such as human hands. Objects are defined as chiral if they exist as a pair of "left-handed" or "right-handed" mirror images that cannot be superimposed (Figure 1). Single-handed chiral organic materials have shown potential or even commercial application in certain electronic devices. For example, the first thermotropic liquid crystalline mesophase discovered was a cholesteric (chiral nematic) phase, based on a chiral cholesterol organic molecule, ${ }^{1}$ which has found extensive application in
}

liquid crystalline display technologies. Despite this, in the area of organic electronic devices, for example, organic light-emitting diodes (OLEDs), organic field-effect transistors (OFETs), and organic photovoltaics (OPVs), the vast majority of organic semiconducting (OSC) materials employed are not chiral (Figure 1). Given the successful and rapid development of organic device technologies, in some cases to the commercial

Received: May 20, 2017

Accepted: July 11, 2017

Published: July 11, 2017 


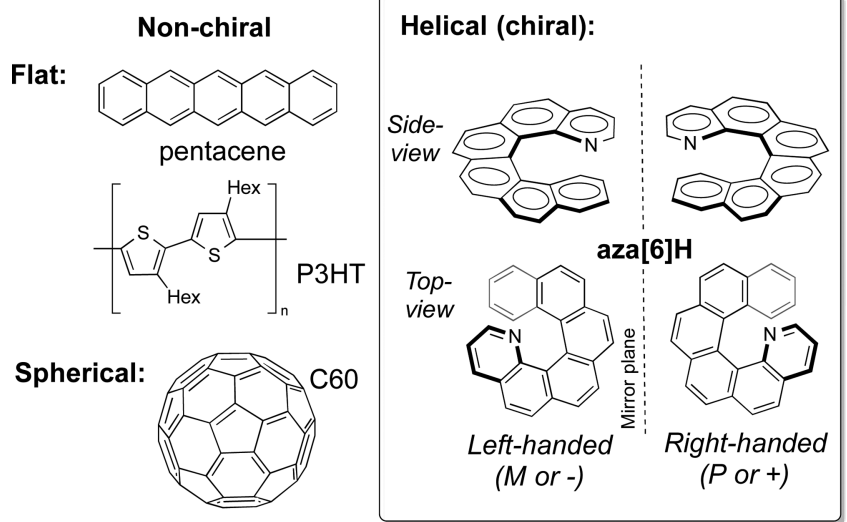

Figure 1. Examples of organic semiconductors, including the chiral 1-aza[6]helicene $(\mathrm{aza}[6] \mathrm{H})$.

marketplace, it could be questioned whether the introduction of molecular chirality into OSCs would add value over conven- tional nonchiral materials (such as those shown in the left-hand side in Figure 1). However, there is increasing recognition in future device applications where the chirality of the organic material is essential for the functionality of the device. ${ }^{2}$ Examples include the use of chiral organic materials as electron spin filters for spintronic applications, ${ }^{3,4}$ as sensors of chiral small molecules, ${ }^{5}$ as circularly polarized (CP) emissive materials, $^{6-11}$ and in CP-photodiodes. ${ }^{12}$ Chirality has also been used as a structural probe, where the assembly and aggregation of device-relevant chiral $\pi$-conjugated small molecules or polymers have been studied using highly sensitive chiroptical spectroscopy. ${ }^{13-15}$ We previously reported the use of enantiopure (single handed) 1-aza[6] helicene $(\mathrm{aza}[6] \mathrm{H}$, Figure 1), an intrinsically helical (and hence chiral) organic semiconductor, in the production of organic photoFETs which could detect and differentiate circularly polarized (CP) light. ${ }^{16}$ This effect was a result of the known strong chiroptical properties of this class of molecules, ${ }^{17,18}$ where left- or righthanded aza[6] $\mathrm{H}$ preferentially absorbs left- or right-handed $\mathrm{CP}$ light and mediates a selective photoresponse.
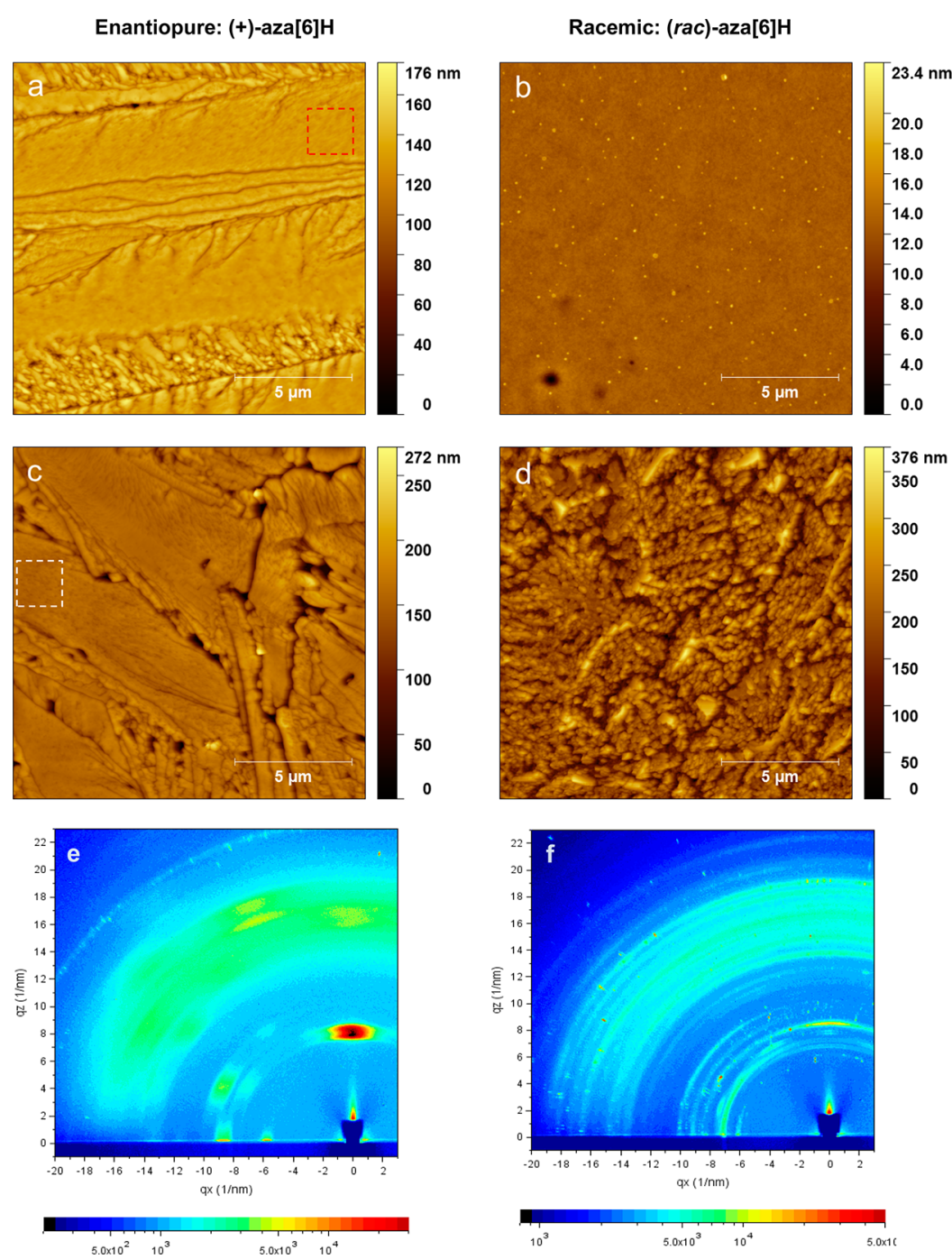

Figure 2. Structural characterization of aza[6]H films. Comparison of thin-film morphology and structure over time for the $(+)$-aza[6]H thin film (left-hand panel) versus the (rac)-aza[6]H thin film (right-hand panel): AFM data for "as cast" films (a, b) and after $24 \mathrm{~h}$ (c, d), scan size of $15 \times 15 \mu \mathrm{m}$ was selected in order to obtain a survey of topologies over a large area. The false-color ruler signifies topographical height maxima and minima for each AFM image, and dashed squares of $2 \times 2 \mu \mathrm{m}$ size (red in (a) and white in (c)) represent the respective typical smooth/glassy regions, from which the RMS value for the smooth region was taken for each case; 2D GIWAXS patterns after $20 \mathrm{~h}$ (e and $\mathrm{f}$ ). 

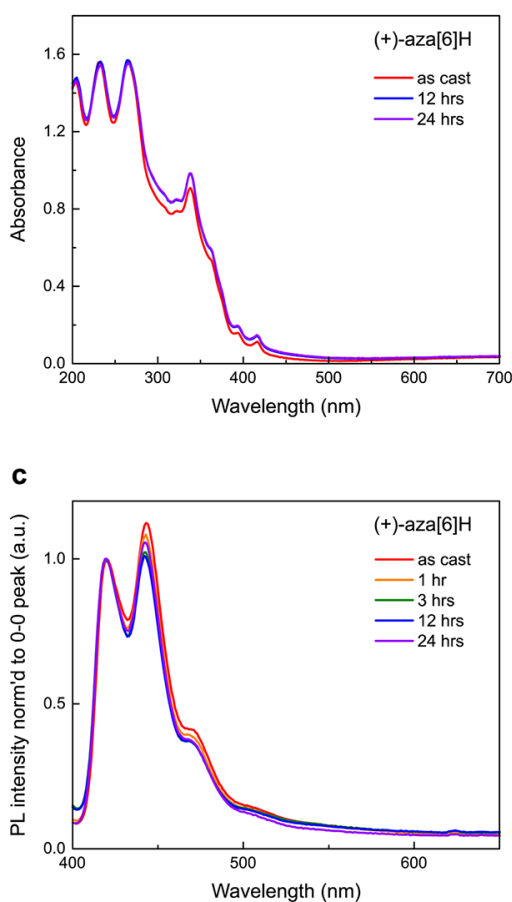

b

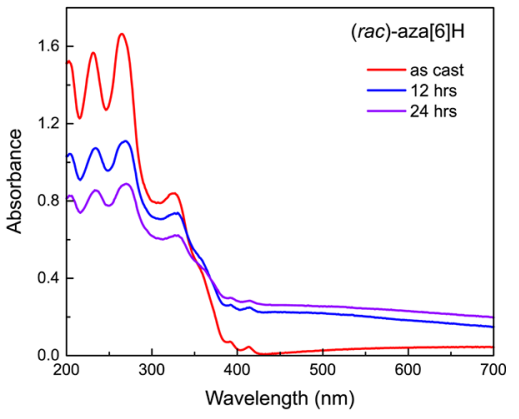

d

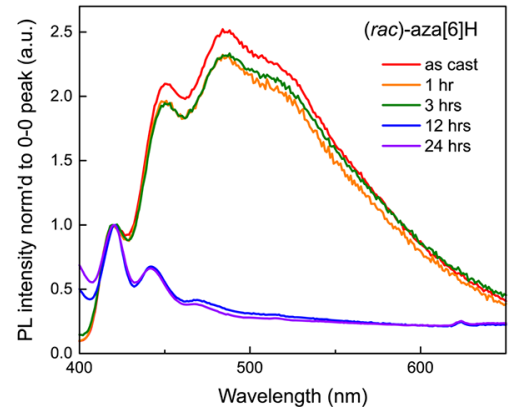

Figure 3. Characterization of the absorption and emission profiles of aza[6]H films. Comparison of absorption (a) and PL (c) spectra for the $(+)$-aza[6] H thin film (left-hand panels) versus their counterparts of the (rac)-aza[6] H thin film (right-hand panel, absorption (b) and PL (d)) over $24 \mathrm{~h}$. The absorption spectra are as-recorded. The PL spectra are normalized to the single-molecule $S_{1} \rightarrow S_{0} 0-0$ transition peak at about $420 \mathrm{~nm}$. The PL excitation wavelength was $380 \mathrm{~nm}$.

It is well-known that the key to successful and highperformance organic devices is the need to link well-understood characteristics of an isolated molecule (so-called "molecular" properties: HOMO/LUMO energy levels, molecular optical properties, etc.) into the collective behavior of multiple units in thin films ("material" properties). ${ }^{19}$ All the examples cited above employ the chiral organic material in an enantiopure (single handed) composition - the material consisting of solely left- or right-handed molecules. However, other chiral compositions could be envisaged using mixtures of the leftand right-handed structures; the most common being a socalled racemate (a 50:50 mixture of left-handed and righthanded molecules). As the right- and left-handed enantiomers of a given chiral material have identical "molecular" properties, these properties would not be expected to change when comparing an enantiopure material to a racemate. However, just like for shaking hands, the interactions between, e.g., two right-handed molecules and a right- and a left-handed molecule are not equivalent, meaning an enantiopure substance and a racemate have different bulk packing and, ultimately, different bulk material properties. The possibility to exploit chirality to alter the "material" properties without affecting the "molecular" properties is a fascinating concept which could lead to wide ranging and currently unexploited possibilities in the area of OSC design and optimization, particularly for usuage in devices.

In this paper, we report a proof-of-concept study with the helicene aza[6] $\mathrm{H}$ (Figure 1), demonstrating how the material and device properties of this OSC differ according to whether the single-handed (enantiopure) (+)-aza[6] $\mathrm{H}$ or the racemic (rac)-aza[6]H composition is employed. Monolayers of a number of helicenes have been studied on a range of metallic surfaces, and it has been shown that their chirality is central to the 2D structures obtained. ${ }^{20-27}$ Coupled with the device compatibility of aza[6]H (see above), ${ }^{7,16}$ we reasoned that a helicene would provide an excellent representative chiral OSC for the study. It is worth noting that studies linking chirality to conductivity are extremely rare. ${ }^{28-31}$ Furthermore, attempts to use the chiral composition of an OSC to tune the conductive properties in the context of organic electronic devices has so far been very limited, ${ }^{32,33}$ with only two recent reports in 2017. The first is a study of P3HT-blend OPVs by Favereau, Blanchard, Cabanetos, Crassous, and co-workers, ${ }^{32}$ who showed a 5-fold increase in power conversion efficiency when switching from a racemic to an enantiopure additive as part of the polymer blend material. The second is a study by Oh and co-workers, ${ }^{33}$ who showed that pendant chirality in selfassembled perylene diimide crystalline nanowires could be used to effect performance in organic phototransistors, where the homochiral aggregates had an improved electron mobility. Although other studies of OPVs ${ }^{34,35}$ or $\mathrm{OFETs}^{36}$ have employed chiral compounds, these were only compared to isomers that are not related by mirror symmetry (so-called diastereoisomers); in effect, a comparison of different compounds with different "molecular" properties. Here, we show how the emergent properties of this aza[6]H are dependent on chiral composition (racemic versus enantiopure) and demonstrate an over 80 -fold increase in the hole mobility in organic field-effect transistors upon switching from a singlehanded to racemic material. The experimental results are supported by detailed crystal structure predictions (rarely used in the study of molecular materials) that confirm the influence of chiral interactions on bulk packing and, thus, bulk charge transport. On the basis of these results, we believe that chiral composition could be a useful means to control the macromolecular organization and electronic properties of 
a)

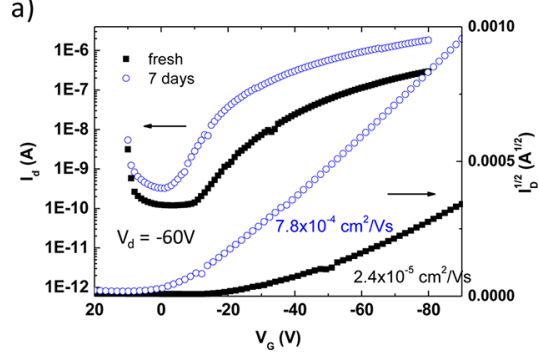

b)

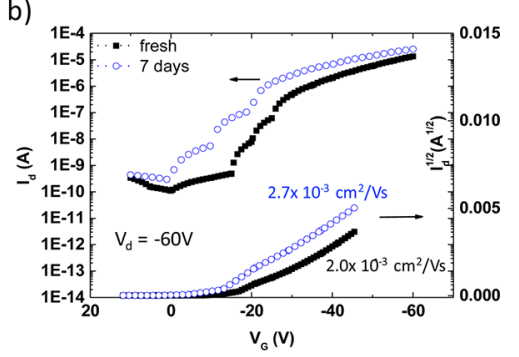

Figure 4. OFET data for the two chiral compositions of aza[6]H. Transfer characteristics of bottom-gate, bottom-contact OFETs in which the active organic semiconductor material is $(\mathrm{a})(+)$-aza $[6] \mathrm{H}$ and $(\mathrm{b})(\mathrm{rac})$-aza[6] $\mathrm{H}$, measured at a source-drain bias of $60 \mathrm{~V}$ directly after device fabrication (filled squares) and after 7 days aging in ambient conditions (open circles). The resulting hole mobilities are indicated for fresh (black text) and aged (blue text) devices.

future (chiral) organic semiconducting materials in organic electronic devices.

\section{RESULTS AND DISCUSSION}

Initially, we investigated the impact of enantiopure versus racemic chiral composition on self-assembly in the solid state. Thin films $(\approx 160 \mathrm{~nm})$ of $(+)$-aza[6] $\mathrm{H}$ and $(\mathrm{rac})$-aza[6] $\mathrm{H}$ on fused silica substrates were prepared via spin coating from solution. With the naked eye, the freshly prepared films of both enantiopure (+)-aza[6] $\mathrm{H}$ and racemic $(\mathrm{rac})$-aza[6] $\mathrm{H}$ present a smooth and glassy state, with high layer transparency and no discernible haze due to scattering of light. Topographies of the deposited films were compared by tapping-mode atomic force microscopy (AFM, Figure 2). As cast $(+)$-aza[6] H thin films were found to be predominantly smooth and amorphous-like, with apparently featureless areas having a typical RMS surface roughness $\approx 3.3 \mathrm{~nm}$ (see highlighted region in Figure $2 \mathrm{a})$. The as cast (+)-aza[6]H layers also feature some "ditch-like" textures across the surface with some (plausibly) crystal domains/grains populated in close proximity. In contrast, as cast $(\mathrm{rac})$-aza [6] $\mathrm{H}$ thin films (Figure $2 \mathrm{~b}$ ) were found to have an ultrasmooth surface with a RMS roughness of $\approx 0.64 \mathrm{~nm}$, despite having vertically standing "columns" with average height of $\approx 8.5 \mathrm{~nm}$. These can probably be associated with initial nucleation and crystallization in the $30 \mathrm{~min}$ delay between film deposition and the AFM scan. After $24 \mathrm{~h}$, the (+)-aza[6]H molecules aggregated to form textures with more pronounced "gullies", along with the coexistence of smooth regions $(\approx 5.8 \mathrm{~nm}$ RMS roughness, e.g., region highlighted by a white, dashed square in Figure 2c) and some more granular regions. For $(\mathrm{rac})$-aza[6]H thin films, a larger extent of molecular self-assembly was observed over time, forming crystallized grain clusters with a significantly increased RMS surface roughness of $\approx 46 \mathrm{~nm}$ (Figure $2 \mathrm{~d}$ ), demonstrating the drastically different temporal evolutions of enantiopure and racemic thin-film surface morphologies. This "microscopic" indication by AFM was supported by the "macroscopic" observation with the naked eye, as the $(\mathrm{rac})$-aza[6] $\mathrm{H}$ thin films became visibly cloudy after $24 \mathrm{~h}$.

To further study how thin-film molecular packing and orientation varies with chiral composition, grazing incidence wide-angle X-ray scattering (GIWAXS) experiments were performed. GIWAXS of the (+)-aza[6]H thin film over time revealed self-assembly kinetics comparable to those observed by AFM, giving well-defined and highly-oriented structures after $20 \mathrm{~h}$ at room temperature (Figure $2 \mathrm{e}$ and Figure S1). Analogous structures to those obtained with $(+)-a z a[6] \mathrm{H}$ were observed for the mirror image enantiomer, $(-)$-aza $[6] \mathrm{H}$, as would be expected (see Figure S2). However, the structures obtained for the enantiomerically pure and racemic compositions were clearly very different (Figure 2e versus $2 \mathrm{f}$ ), again in agreement with the AFM data in Figure 2c,d.

Based on these morphological studies, we performed photophysical measurements with comparable time delays after film deposition. As may be expected, freshly deposited thin $(160 \mathrm{~nm})$ films of $(+)$-aza[6] $\mathrm{H}$ and $(\mathrm{rac})$-aza[6] $\mathrm{H}$ have similar absorption characteristics in the UV spectral region from 200 to $400 \mathrm{~nm}$ (Figure 3a,b), which correspond well to the solutionstate spectrum. ${ }^{16}$ For $(+)$-aza $[6] \mathrm{H}$, the absorption spectra are almost independent of the morphological changes observed over time, with sound spectral overlap between the data obtained for freshly prepared thin films and those obtained after $24 \mathrm{~h}$. For ( $\mathrm{rac}$ )-aza[6] $\mathrm{H}$, the absorption decreased with time (Figure $3 \mathrm{~b}$ ). The background between approximately 450 and $700 \mathrm{~nm}$ also increased with time, indicative of an increase in scattering; this was accompanied by a transition from a clear to a more opaque and hazy film. The crystallite sizes and orientations which likely cause this scattering do not form in the (+)-aza[6]H films over the same time scales, again indicative of the difference in enantiopure and racemic morphological behavior.

In terms of emission, freshly prepared $(+)$-aza[6] $\mathrm{H}$ thin films were found to exhibit photoluminescence $(\mathrm{PL})$ in the violet region of the spectrum, with the $S_{1} \rightarrow S_{0} 0-0$ transition at 420 $\mathrm{nm}$ and vibrational sidebands at about 445,470 , and $510 \mathrm{~nm}$ (Figure 3c). This resembles the PL emission spectrum in solution (Figure S3) and can therefore be attributed to an isolated single-molecule vibronic transition. The intensity of the vibrational sidebands slightly decreases relative to the $0-0$ transition with time, which can be related to changes in molecular ordering as the film crystallizes. The fresh ( $\mathrm{rac}$ )aza[6]H thin films instead exhibit a broad emission band (Figure 3d) between 410 and $600 \mathrm{~nm}$ with distinct peaks at about 450,480 , and $515 \mathrm{~nm}$; there is an additional weaker band at $420 \mathrm{~nm}$, which can be associated with the 0-0 singlemolecule transition. The broad emission band disappears completely over $24 \mathrm{~h}$ as the films crystallize and is replaced with only the solution-like spectrum; although the difference in relative $0-0$ transition and sideband intensity compared to the enantiopure films indicates a difference in molecular packing. We suggest the broad emission band may originate from an exciplex involving a molecular aggregate formed between the right- and left-handed enantiomers; no such broad band is observed for the enantiopure material. Furthermore, the broad emission only occurs for the particular intermolecular orientations and distances formed when the racemic thin 

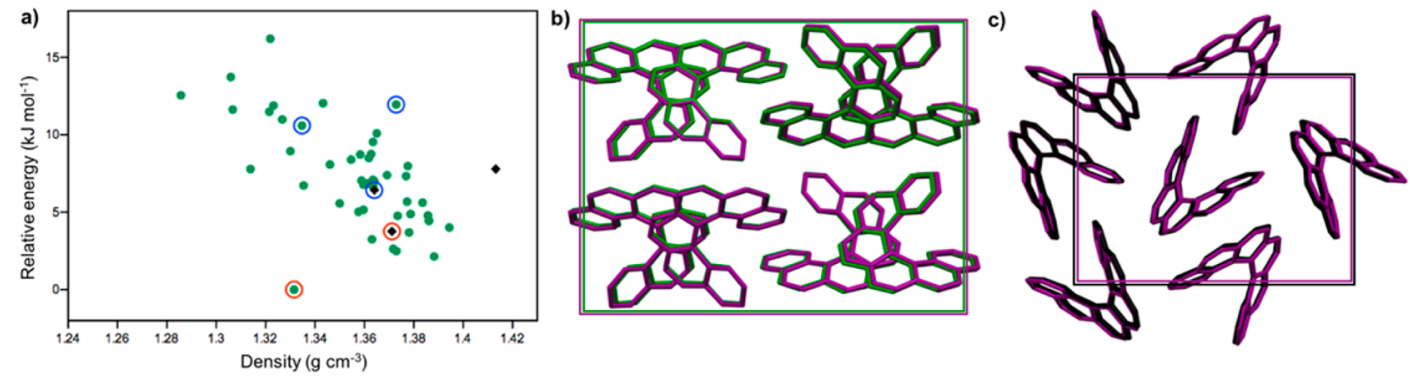

d)

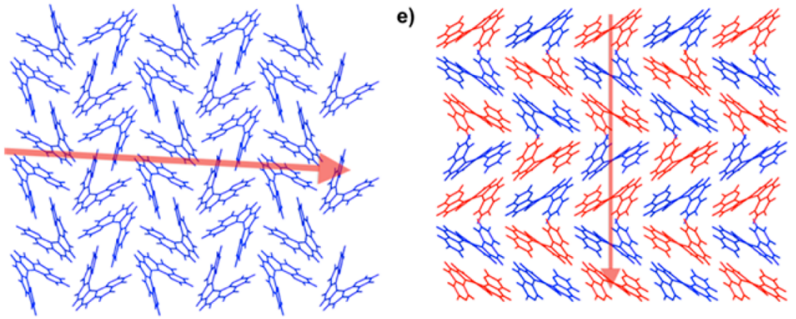

f)

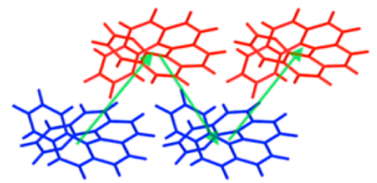

Figure 5. Computational analysis of bulk assembly and transport for aza[6] H chiral compositions. (a) Lattice energy rankings from the crystal structure prediction rationalize the bulk crystal structures of aza[6]H. Chiral packings (black diamonds) and racemate packings (green circles) are shown, with the reported single-crystal bulk chiral and racemate structures indicated with red circles and the possible film structures indicated with blue circles. (b) An overlay of the observed bulk crystal structure (purple) ${ }^{37}$ and the predicted lowest-energy (rac)aza[6] H structure (green). The root-mean-square deviation in the atomic positions of a cluster of 15 molecules, excluding hydrogen positions, RMSD $_{15}$, is $0.180 \AA$. (c) An overlay of the single-crystal structure ${ }^{38}$ (purple) and predicted lowest-energy (+)-aza[6]H structure (black). The RMSD $_{15}$ is $0.351 \AA$. (d) The assigned (+)-aza[6] H thin-film structure in the $b-c$ plane. (e) The lowest-energy assigned (rac)-aza[6]H thin-film structure in the $a-c$ plane, with molecules color coded (blue, left-handed; red, right-handed). Red arrows on the structures depict the direction of hole transport with the highest mobility. (f) Detail of the route of the highest hole mobility in $(\mathrm{rac})$-aza[6] $\mathrm{H}$, where the high coupling pathway is between molecules of alternating handedness.

films are first deposited. Once self-organization occurs and the molecules pack into the crystallized state, the PL returns to that of the single-molecule-type emission.

The studies above suggest that the chiral composition of aza $[6] \mathrm{H}$ has a fundamentally important impact on molecular assembly in the condensed state, which subsequently impacts upon the film morphology and photophysics. Previously, we have reported the use of $(+)-\mathrm{aza}[6] \mathrm{H}$ and $(-)$-aza[6] $\mathrm{H}$ as the OSC in a circularly polarized (CP) light-detecting photoFET and found that the material was solution processable and gave rise to well-behaved OFET characteristics. ${ }^{16}$ Bottom-gatebottom-contact OFETs were fabricated from (+)-aza[6] $\mathrm{H}$ and ( $\mathrm{rac})-\mathrm{aza}[6] \mathrm{H}$ in order to compare how chiral composition impacts hole mobility in a device setting. OFETs fabricated from both (+)-aza[6] H and ( $\mathrm{rac})$-aza[6] $\mathrm{H}$ exhibited good output and transfer characteristics (Figure S4). Notably, hole mobility in the freshly prepared devices differed by over 80 -fold (Figure 4): (+)-aza[6] $\mathrm{H}$ has a hole mobility of $2.4 \times 10^{-5} \mathrm{~cm}^{2} /$ $(\mathrm{V} \mathrm{s})$, whereas $(\mathrm{rac})$-aza[6] $\mathrm{H}$ has a mobility of $2.0 \times 10^{-3} \mathrm{~cm}^{2} /$ (V s). Given the polycrystalline nature of the aza[6] $\mathrm{H}$ thin films (Figure 2), it is likely that both bulk molecular ordering within the crystalline grains and the chiral composition-dependent differences in crystal grain packing, crystallite domain size, etc. are responsible for these observed effects. Consistent with the structural and spectroscopy studies above, devices fabricated from both $(+)$-aza[6] H and $(\mathrm{rac})$-aza[6]H show an enhancement in device mobility over time-a product of the timedependent ordering process-although the effect is more pronounced for $(+)$-aza[6]H. Specifically, (+)-aza[6]H OFETs show an increase in mobility from $2.4 \times 10^{-5} \mathrm{~cm}^{2} /(\mathrm{V} \mathrm{s})$ to 7.8 $\times 10^{-4} \mathrm{~cm}^{2} /(\mathrm{V} \mathrm{s})$ (a 30-fold increase) after 7 days under ambient conditions. OFETs from ( rac)-aza[6]H show a more modest 1.4 -fold increase over the same time period. OFET mobility depends on charge transport within a $2-3 \mathrm{~nm}$ thick helicene layer deep at the buried dielectric-molecular semiconductor interface. The fact that mobility changes observed for aza[6]H over time are slower than those observed by structural and spectroscopic techniques may reflect the fact that the interfacial molecular rearrangement deep in the film is a slower process than that which results in the observed spectroscopic effects (Figure 3). We note that such mobilities in (+)-aza[6]H OFET devices are suitable for CP light detecting applications. ${ }^{16}$

Given the morphological, spectroscopic, and device differences observed between the enantiopure and racemic chiral compositions of aza[6] H, we sought to identify the molecular assembly present in the thin films and compute directiondependent mobility in such films, in order to rationalize the effects observed. The GIWAXS data for the final assembled $(>20 \mathrm{~h}$ post- spin coating) thin-film structures (Figure $2 \mathrm{e}, \mathrm{f}$ ) were compared to the X-ray diffraction patterns expected for the bulk assemblies identified in the single-crystal structures of the two aza[6]H chiral compositions studied. ${ }^{37,38}$ For ( rac)aza[6]H (CCDC 664670), diffraction pattern analysis showed that the thin-film packing was not consistent with that observed in the published ${ }^{37}$ single-crystal structure (Figure S5). Likewise, for enantiopure (+)-aza[6]H (CCDC 921948), the singlecrystal structure $^{38}$ was also not a good fit for the thin-film GIWAXS data (Figure S6). Differences in molecular packing between thin films and single crystals (which are also potentially subject to polymorphism) are not uncommon. ${ }^{39}$

In order to survey alternative bulk structures for thin films of racemic and enantiopure aza[6]H, we conducted a crystal structure prediction (CSP) study. ${ }^{40}$ CSP is an approach developed for the prediction of polymorphism in pharmaceutical molecules, which, although rarely applied thus far in the 
field of molecular materials, ${ }^{41}$ may offer opportunities for rational design of materials. CSP involves global lattice energy minimization, with ideally only the molecular structure required as an input for the calculations. As the crystal packing in aza[6] $\mathrm{H}$ is a result of relatively subtle differences in intermolecular interactions, mostly $\mathrm{C}-\mathrm{H} \cdots \pi$ interactions, we performed an optimization of the lowest 50 hypothetical polymorphs with density-functional theory (DFT) and the exchange-hole dipole moment ${ }^{42}$ (XDM) model of dispersion. The XDM method has previously been shown to perform well for the relative energies of chiral crystals ${ }^{43}$ and therefore is most appropriate for this case. An accurate description of the intermolecular interactions has been shown to be important for effective energetic ranking of hypothetical polymorphs in blind crystal structure prediction tests. ${ }^{4,45}$

We performed CSP on (+)-aza[6] H and (rac)-aza[6]H and observed interesting chiral composition-dependent trends. For example, only three low-energy (+)-aza[6] H polymorphs were found within $15 \mathrm{~kJ} \mathrm{~mol}^{-1}$ of the global minimum, whereas more than 45 low-energy $(\mathrm{rac})$-aza[6] $\mathrm{H}$ polymorphs were identified in the same energy window (Figure 5a). While both (+)-aza[6]H and (rac)-aza[6] $\mathrm{H}$ polymorphs exhibited C$\mathrm{H} \cdots \pi$ interactions between helicene pairs, the low-energy polymorphs were not isomorphous to each other. Both the lowest-energy hypothetical (+)-aza[6]H packing and the lowest-energy hypothetical $(\mathrm{rac})$-aza[6] $\mathrm{H}$ packing were found to match the experimental single-crystal X-ray diffraction structures (Figure $5 \mathrm{~b}, \mathrm{c}$ ). The racemic packing was found to be $\approx 3.8 \mathrm{~kJ} \mathrm{~mol}^{-1}$ lower in energy than the chiral $(+)$-aza $[6] \mathrm{H}$ packing. This successful prediction of both the $(+)-a z a[6] \mathrm{H}$ and ( $\mathrm{rac})$-aza[6] $\mathrm{H}$ observed crystal structures demonstrates the potential for CSP to assist in the design of OSCs from molecular structure to bulk properties by bridging the gap between molecular and bulk structure.

Through comparison of the 50 lowest-energy simulated CSP structure XRD diffraction patterns with the measured GIWAXS data on aged films, we were able to identify likely polymorphs and orientations for molecular packing in the thin films of (rac)-aza[6] $\mathrm{H}$ and (+)-aza[6] H (Figure 5). For further detailed discussion of this assignment, see the SI. For $(\mathrm{rac})-\mathrm{aza}[6] \mathrm{H}$, two potential matches were identified for the thin-film structure: a lower-energy structure which was $10.6 \mathrm{~kJ} \mathrm{~mol}^{-1}$ above the global minimum (Figure S7); and a slightly higherenergy structure which was $11.9 \mathrm{~kJ} \mathrm{~mol}^{-1}$ above the global minimum (Figure S8). Based on these assignments and analysis of the GIWAXS data, it is also apparent that several orientations of the unit cell relative to the substrate are present in the experimental $(\mathrm{rac})$-aza[6] $\mathrm{H}$ thin film. For $(+)-\mathrm{aza}[6] \mathrm{H}$, one of the simulated CSP polymorphs-predicted to be the next lowest in energy after the observed single-crystal polymorph-was found to give a good match to the GIWAXS data (Figure S9), once the $a$-axis was doubled. This assigned structure is consistent with the thin-film crystallites in the experimentally studied $(+)$-aza[6] $\mathrm{H}$ thin film being preferentially oriented in the same direction, with the (lll $\left.\begin{array}{ll}1 & 0\end{array}\right)$ plane orientated parallel (and the unit cell $a$-axis aligned close to normal) to the substrate plane (Figure 5c).

Using the lowest-energy assigned thin-film polymorph for the $(\mathrm{rac})$-aza[6] $\mathrm{H}$ and the assigned $(+)-\mathrm{aza}[6] \mathrm{H}$ polymorph, we computed average and orientation-dependent hole mobility for each structure to gain further insight into the charge-carrier mobilities possible for macroscopic single crystals of the two chiral compositions studied. Briefly, the orientation-dependent hole mobility is calculated in the weak-coupling limit for each crystal structure, by calculating hole transfer integrals between interacting molecules, evaluating charge-transfer rates using nonadiabatic Marcus theory, and then solving a master equation for the hole velocity in response to the electric field $^{46}$ (see Methods). The largest nearest-neighbor transfer integrals were 39 and $52 \mathrm{meV}$ for $(+)$-aza[6] $\mathrm{H}$ and $(\mathrm{rac})$-aza[6] $\mathrm{H}$ structures, respectively, supporting the use of the weak coupling approximation. We use a reorganization energy of 470 $\mathrm{meV}$ based on a calculated inner component of $0.17 \mathrm{eV}$ and estimated outer component of $0.3 \mathrm{eV}$. The magnitude of the reorganization energy will influence the magnitude of the calculated mobilities but not the relative values. The average mobility calculated for $(\mathrm{rac})$-aza[6] $\mathrm{H}$ was $0.058 \mathrm{~cm}^{2} \mathrm{~V}^{-1} \mathrm{~s}^{-1}$, while the average for (+)-aza[6] $\mathrm{H}$ was $0.032 \mathrm{~cm}^{2} \mathrm{~V}^{-1} \mathrm{~s}^{-1}$. These mobilities are predicted to be dependent on the direction of transport (see Figures S11 and S12 for polar plots). For the (+)-aza[6] H structure, the largest predicted hole mobility $\left(0.052 \mathrm{~cm}^{2} \mathrm{~V}^{-1} \mathrm{~s}^{-1}\right)$ was observed to be in the $b-c$ plane (Figure $5 \mathrm{~d}$ ), whereas for the (rac)-aza[6] H structure, the largest mobility $\left(0.14 \mathrm{~cm}^{2} \mathrm{~V}^{-1} \mathrm{~s}^{-1}\right)$ was found for a direction in the $a-b$ plane (Figure 5e). Interestingly, this high mobility of $(\mathrm{rac})-\mathrm{aza}[6] \mathrm{H}$ is enabled by a hole transfer route between molecules of alternating handedness (see Figure 5f), a situation that can only occur in racemic crystals.

Clearly the calculated mobility values demonstrate that the different bulk crystal structures obtained for the racemic versus enantiopure aza[6] $\mathrm{H}$ thin films lead to different intrinsic charge transport, both in terms of average and orientation-dependent hole mobility. The calculations, using the crystal structures identified with the aged samples, reproduce the trend observed in the aged OFET devices, namely that the calculated hole mobility is larger (by a factor of $\sim 2$ on average and $\sim 3$ for the maximum values) for the $(\mathrm{rac})$-aza[6] $\mathrm{H}$ than the $(+)-\mathrm{aza}[6] \mathrm{H}$ structure, comparable with the experimental ratio of a factor of $\sim 3$. However, other effects also contribute to the actual mobility observed in OFETs fabricated from polycrystalline OSCs, including crystallite size, intercrystallite distance, crystallite orientation relative to the substrate and contacts, the presence of traps at the grain boundaries, and dielectricsemiconductor interface and contacts. While the maximum OFET hole mobilities observed experimentally are more than an order of magnitude lower than the calculated single-crystal mobility values, this difference can be assigned in part to the polycrystalline structure of the experimental thin films, as observed in the AFM images (Figure 2). Large differences of 1-2 orders of magnitude between single-crystal and polycrystalline experimental mobility values are typical for molecular semiconductor transistors, ${ }^{47-49}$ in which the ratelimiting step in charge transport is transfer between the crystalline grains. Taken together, it would seem that for aza[6]H, a racemic composition gives rise to many more possible low-energy polymorphs, from which a thin-film structure can be obtained with higher calculated and experimental hole mobility. Thus, chiral composition alone can clearly strongly impact structure and transport in this helical OSC.

\section{CONCLUSIONS}

We have shown that by solely varying the chiral composition of the helically chiral OSC aza[6] $\mathrm{H}$ between enantiopure and racemic, significant changes in morphology, photophysics, and device performance can be observed. Our results are consistent 
with those of Nakamura and co-workers, who previously showed that sublimed films of azaboradibenzo[6]helicene exhibit differential photoinduced charge transport (measured using time-of-flight (TOF) measurements) depending on whether racemic or enatiopure material is employed. ${ }^{28}$ Taken together, this suggests that chiral composition could represent a currently unexploited means of tuning the properties of an OSC of a given "molecular" composition. Currently, the central approach in the design of organic devices with differential electronic properties is to vary the chemical structure of OSC materials, which often requires the reoptimization of the precise device architecture to ensure it is compatible with the OSC(s) selected. Because the "molecular" properties of mirror image (enantiomeric) forms of a chiral material are exactly the same, the use of chiral composition to control electronic properties provides a potentially much simpler way in which one could alter material functionality without affecting device compatibility. This would allow high versatility in matching processing conditions to device architecture and device function; a paradigm shift in the way OSC materials and devices are traditionally approached. Thus, we believe the further study and application of chiral OSC materials should lead to a rich area of science in organic electronic device architectures. While we have only focused on two chiral compositions in this studyracemic and enantiopure-one could envisage using compositions containing an unequal mix of left- and right-handed molecules (a so-called scalemic mixture). Scalemic material may give further unexpected results, dependent on its chiral composition (enantiomeric ratios from 51:49 to 99:1) and thus should provide fascinating insight on the role of chiral composition in structure and charge transport. For example, scalemic material may phase separate into homochiral domains of opposite handedness ${ }^{20}$ or separated homochiral and heterochiral domains, each with attendant changes in bulk structure and mobility.

Thus, although this study provides proof-of-concept results as to how chiral composition can be used to impact morphological, spectroscopic, and device properties for conjugated organic materials, there is clearly scope for significant development in the approach. The combination of CSP, a technique underutilized in organic material discovery and optimization, together with charge-transport predictions should allow for the rational "bottom up" design of chiral OSCs, which may allow for the identification of chiral polymorphs with intrinsically large mobility values and/or organic materials with large differential mobility between chiral compositions. Furthermore, the large orientation dependency predicted for charge transport within our chiral materials suggests that strategies (chemical design, fabrication techniques) to control orientation of a given polymorph relative to the substrate could lead to dramatically improved, chiral composition-dependent charge-transport mobilities.

\section{METHODS}

Aza[6]helicene was synthesized as previously reported ${ }^{50}$ and separated into (+)-1-aza[6]helicene and (-)-1-aza[6] helicene by preparative chiral HPLC by Chiral Technologies Europe using a CHIRALPAK ID $5 \mu \mathrm{m}, 250 \times 30 \mathrm{~mm}$; carbon dioxide/ethanol $70 / 30 ; 120 \mathrm{~mL} / \mathrm{min}$; detection UV $350 \mathrm{~nm}$; outlet pressure $150 \mathrm{bar}$; temperature $25{ }^{\circ} \mathrm{C}$; retention time $(+)-\mathrm{aza}[6] \mathrm{H} 7.1 \mathrm{~min} ;(-)-\mathrm{aza}[6] \mathrm{H} 10.2 \mathrm{~min}$; both fractions $>99.5 \%$ ee.

Helicene Thin-Film Deposition. $20 \mathrm{mg} \mathrm{mL}^{-1}$ solutions of enantiopure and racemic helicenes were prepared in HPLC-grade toluene. Freshly prepared solutions were kept stirring for $3 \mathrm{~h}$ at $50{ }^{\circ} \mathrm{C}$ to ensure homogeneous dissolution. Before film deposition, solutions were filtered using $0.45 \mu \mathrm{m}$ pore size PTFE membrane filters to remove any potentially undissolved molecule aggregates and dust/ particulates. Thin-film deposition was achieved by spin coating solutions at $1200 \mathrm{rpm}$ for $60 \mathrm{~s}$, onto fused-silica substrates cleaned via sequential acetone, isopropanol ultrasonic baths and UV-ozone treatment. An average thin-film thickness of $160 \mathrm{~nm}$ was measured by DektakXT profilometer.

Photophysical and Morphology Characterization. Absorption and PL spectra of enantiopure and racemic helicene thin films were measured using a Shimadzu UV-2550 UV-vis spectrophotometer and a Horiba Jobin Yvon FluoroMax-3 spectrofluorometer, respectively. Absorption measurements were performed in transmission mode UVvis only; this is with the T\% of the bare fused silica substrate accounted for, but without further correction of the specular or diffuse reflectance of the films. Solution-phase PL spectra are uncorrected for detector response and were measured on a FluoroMax-4 (Horiba Jobin Yvon), using freshly prepared chloroform solutions that were deoxygenated by sparging for $15 \mathrm{~min}$ with a stream of chloroform-saturated nitrogen. Thin-film surface morphological studies were carried out in tapping mode using an Agilent Technologies 5500 AFM system in the dark under ambient air. A scan area of $15 \times 15 \mu \mathrm{m}$ was selected in order to obtain surveys of the topography over a large area. The "as cast" AFM images were fully acquired (i.e., scan completed) $\sim 30 \mathrm{~min}$ after the initial thin-film deposition due to intervals of sample transfer and the time required for each AFM measurement scan. To investigate the dynamic development of the self-assembling in helicenes, photophysics and morphology characteristics were initially measured on freshly coated samples which were then kept in nitrogen atmosphere and measured at set intervals as shown in figures in the main text.

GIWAXS Experimental Details. Measurements were performed at the D1 station at the Cornell High Energy Synchrotron Source (CHESS). A bending magnet source in combination with multilayer optics supplied an intense X-ray beam with a wavelength of $0.115 \mathrm{~nm}$ and $1.5 \%$ bandwidth. The X-ray beam was collimated to a size of 0.5 $\mathrm{mm}$ horizontally and $0.1 \mathrm{~mm}$ vertically using a slit system. Films prepared as above were placed on a 5-axes goniometer and lined up such that the incident beam impinged on the sample at a typical angle of $0.15^{\circ}$. Scattered X-rays were detected with a MedOptics CCD camera with a pixel size of $46.9 \mu \mathrm{m}$, placed $101 \mathrm{~mm}$ downstream from the sample. The intense reflected beam was blocked with a $1.5 \mathrm{~mm}$ molybdenum rod midway between sample and camera. At an effective camera size of $45 \mathrm{~mm}$, the camera could register X-rays with wave vectors up to $20 \mathrm{~nm}^{-1}$ corresponding to minimum $d$-spacings of 0.3 $\mathrm{nm}$. The camera was calibrated using a cerium oxide standard.

OFET Fabrication and Characterization. Bottom-gate, bottomcontact helicene racemic and enantiomeric OFETs were fabricated by spin-coating a $150 \mathrm{~nm}$ thin film (from a $15 \mathrm{mg} / \mathrm{mL}$ toluene solution) onto prepatterned $\mathrm{Au}$ contact $\mathrm{SiO}_{2} / \mathrm{Si}$ wafer transistor substrates with channel lengths $L$ and widths $W$ of $10 \mu \mathrm{m}$ and $1 \mathrm{~cm}$, respectively. The thickness of the $\mathrm{SiO}_{2}$ insulating layer is $230 \mathrm{~nm}$. For good device performance, a self-assembled monolayer (SAM) of octadecyltrichlorosilane (OTS, Sigma-Aldrich) was deposited onto the surface of the wafer through vapor deposition, and the gold source and drain electrodes were treated with a pentafluorobenzene thiol SAM (PFBT, Sigma-Aldrich) to reduce the contact resistance. The output and transfer characteristics were recorded by an Agilent 4156C semiconductor parameter analyzer in nitrogen atmosphere. Saturation mobility $\mu$ and threshold voltage $V_{\mathrm{T}}$ were extracted from the saturation regime transfer characteristics at $V_{\mathrm{D}}$ of $-60 \mathrm{~V}$ using $I_{\mathrm{D}}=\mu\left(C_{\mathrm{i}} W /\right.$ $2 L)\left(V_{\mathrm{G}}-V_{\mathrm{T}}\right)^{2}$, where $V_{\mathrm{D}}, V_{\mathrm{G}}, I_{\mathrm{D}}$, and $C_{\mathrm{i}}$ are the drain voltage, gate voltage, drain current, and insulator capacitance per unit area, respectively.

Crystal Structure Prediction (CSP). First we constructed a model of the aza[6] $\mathrm{H}$ and geometry optimized the structure in Gaussian $09^{51}$ at the B3LYP/6-31G(d,p) level of theory. CrystalPredictor ${ }^{52,53}$ was then used for global lattice energy minimization calculations on the whole aza[6] $\mathrm{H}$ molecule as the asymmetric unit, with the molecule held rigid. The search was carried out in $P 1, P 2_{1}, P 2_{1} 2_{1} 2_{1}, P 2_{1} 2_{1} 2$, $P 4_{1} 2_{1} 2, R 3, C 2$ and $C 222_{1}, P 2_{1} / c, P-1, C 2 / c, P b c a, P n a 2_{1}, P n m a, P c a 2_{1}$, 
$\mathrm{Pbcn}, \mathrm{Pc}, \mathrm{Cm}, \mathrm{C} m \mathrm{C}_{1}, \mathrm{Aba2}, \mathrm{Fdd2}$ Iba2, Pnna, Pccn, Pbcm, Pnnm, Pmmn, Cmcm, Cmca, Fddd, Ibam, Pa-3, P2 $1 / m, \mathrm{C} 2 / m, P 2 / c, P 4 / n$, $P 42 / n, I 4 / m, I 41 / a, R 3 c, R-3 c, P 63 / m, P-42{ }_{1} c, I-42 d, I-4, C c, P 41, P 43$, $P 4_{1} 22_{1} 2, P 4_{3} 212, P 3_{1}, P 3_{2}, P-3, R-3, P 3_{1} 2_{1}, P 322_{1}, P 61, P 63$, and $P 213$ space groups. This includes both chiral and racemic crystal structures. The global optimization was continued until no new structures were being identified; this corresponded to the determination of approximately 500,000 structures. The structures found in the global lattice calculations were then lattice energy minimized using DMACRYS,${ }^{54}$ with intermolecular interactions taken from the repulsion-dispersion potential $\mathrm{W} 99^{55}$ and the electrostatics from distributed multipole analysis ${ }^{56}$ (calculated using GDMA2 $)^{57}$ of the $\mathrm{MP} 2 / 6-31 \mathrm{G}(\mathrm{d}, \mathrm{p})$ wave function.

Density-Functional Theory Refinement of Hypothetical Polymorphs from CSP. The 50 lowest-energy candidate structures obtained with the W99 potential were selected for additional refinement. These crystal geometries were relaxed using the B86bPBE density functional ${ }^{88,59}$ with the exchange-hole dipole moment (XDM) dispersion correction. ${ }^{60,61}$ A plane-wave/pseudopotentials approach in the framework of the projector augmented wave (PAW) formalism ${ }^{62}$ was used, as implemented in Quantum ESPRESSO, version $5 .^{63}$ The geometry relaxations were carried out with a "tight" convergence criteria $\left(10^{-5}\right.$ energy threshold). For the electronic steps, a $2 \times 2 \times 2 k$-point grid, and cutoff energies of $60 \mathrm{Ry}$ for the plane-wave expansion and $600 \mathrm{Ry}$ for the density expansion were used. The parameters for the XDM damping function were $a 1=$ 0.6512 and $a_{2}=1.4633 .{ }^{64}$ The energy of the isolated molecule, necessary for calculation of the lattice energies, was computed using a cubic supercell with side lengths of 50 Bohr.

Calculations of Hole Mobility. We relaxed the geometry of each molecule in vacuum, with hybrid density-functional theory (B3LYP with a $6-31 G^{*}$ basis set). These geometries we projected back onto the crystallographic positions of the unit cell by the space group symmetry operations and translated by the lattice units to form a $3 \times 3 \times 3$ supercell. We calculate the electronic transfer integral between each molecule in the unit cell to all other molecules in the supercell, subjected to a distance cutoff of $18 \AA$. The transfer integrals were calculated with a hybrid DFT calculation and the projective method, on a molecular pair in vacuum. This involves projecting the orbitals of the pair onto a basis set defined by the unperturbed orbitals of the individual molecules. We use the frozen-orbital approximation, choosing the eigenvector associated with the highest occupied Kohn-Sham orbital on the isolated molecules to represent our initial and final states. The orbital energies of the pair of molecules are then used to rewrite the single electron operator (Fock matrix) in the new localized basis set, ${ }^{65}$ which is then diagonalized to arrive at the hole transfer integral, $J$. This is also known as the hopping integral and represents the kinetic energy of the electron tunnelling between these sites.

The Marcus equation for hopping rates between sites $i$ and $j$ is

$$
\Gamma_{i j}=\frac{\left|J_{i j}\right|^{2}}{\hbar} \sqrt{\frac{\pi}{\lambda k_{\mathrm{B}} T}} \exp \left(-\frac{\left(\Delta E_{i j}+\lambda\right)^{2}}{4 \lambda k_{\mathrm{B}} T}\right)
$$

where $J_{i j}$ is the transfer integral between sites $i$ and $j, \lambda$ is the reorganization energy, and $\Delta E_{i j}$ is the energy difference between sites $i$ and $j$. The reorganization energy is the energy cost associated with the rearrangement of the molecules involved in the hop and their surroundings during charge transfer. The reorganization energy is a sum of the inner $\left(\lambda_{i}\right)$ and outer $\left(\lambda_{0}\right)$ reorganization energies, which refer to the molecules and their surroundings, respectively. The inner reorganization energy was calculated with the four-point method, in a vacuum hybrid DFT calculation: ${ }^{66}$

$$
\lambda_{i}=E_{\text {neutral }}^{+}-E_{\text {charged }}^{+}+E_{\text {neutral }}^{0}-E_{\text {charged }}^{0}
$$

where $E^{+}$and $E^{0}$ are the total energies of the charged and neutral molecules, respectively, and neutral and charged indicate that the energy is computed in the ground-state geometry of the neutral or charged molecule. We calculated the inner reorganization energy to be
$0.171 \mathrm{eV}$. The outer reorganization energy, $\lambda_{0}$, is more difficult to quantify, so we approximated it as $0.3 \mathrm{eV}$. As $J \ll \lambda$, the value of $\lambda$ will scale the overall mobility, while not affecting the relative trends.

The change in energy between the electron being localized on $i$ and $j$ is

$$
\Delta \mathrm{E}_{i j}=\varepsilon_{i}-\varepsilon_{j}-q \overrightarrow{\mathbf{F}} \cdot \overrightarrow{\mathbf{r}}_{i j}
$$

where $\varepsilon_{i}$ and $\varepsilon_{j}$ are the energies of sites $i$ and j, respectively, $q$ is the charge on an electron, $\overrightarrow{\mathbf{F}}$ is the field vector, and $\overrightarrow{\mathbf{r}}_{i j}$ is the distance vector between sites $i$ and $j$.

We construct a Master equation from the microscopic rates: ${ }^{67}$

$$
\frac{d P_{j}}{d t}=\sum_{i \neq j}\left[\Gamma_{i j} P_{i}(t)-\Gamma_{j i} P_{j}(t)\right]
$$

where $P_{j}$ is the probability of site $j$ being occupied and $\Gamma$ is the rate of hopping between sites $i$ and $j$. This equation is valid for the low charge limit. We consider a matrix $A$ with

$$
A_{i j}= \begin{cases}\Gamma_{i j}, & i \neq j \\ -\sum_{i \neq j} \Gamma_{i j}, & i=j\end{cases}
$$

Solving the master equation in the steady state thus becomes equivalent to solving the null-space of

$$
\mathbf{A} P=0
$$

We use the steady-state occupation probabilities so derived to find the average velocity of charges

$$
\vec{v}=\sum_{i \neq j} \vec{r}_{i j} A_{i j} P_{j}
$$

and find the mobility with

$$
\mu=\frac{\langle v\rangle}{F}
$$

where $\langle v\rangle$ is the average velocity of charges in the direction of a field and $F$ is the magnitude of the applied electric field.

\section{ASSOCIATED CONTENT}

\section{Supporting Information}

The Supporting Information is available free of charge on the ACS Publications website at DOI: 10.1021/acsnano.7b03540.

Detailed experimental procedures, thin-film and device fabrication and characterization, GIWAXS data, computational details (PDF)

\section{AUTHOR INFORMATION}

\section{Corresponding Authors}

*E-mail: alasdair.campbell@imperial.ac.uk.

*E-mail: m.fuchter@imperial.ac.uk.

ORCID

Jochen R. Brandt: 0000-0001-7082-6201

Gordon J. Hedley: 0000-0002-1825-6296

Erin R. Johnson: 0000-0002-5651-468X

Kim E. Jelfs: 0000-0001-7683-7630

Matthew J. Fuchter: 0000-0002-1767-7072

\section{Notes}

The authors declare no competing financial interest.

Raw data files from this study are openly available at DOI: 10.6084/m9.figshare.c.3829771. 


\section{ACKNOWLEDGMENTS}

The authors thank the Engineering and Physical Sciences Research Council for funding (EP/I014535/1, EP/L014580/1, $\mathrm{EP} / \mathrm{P} 000525 / 1, \mathrm{EP} / \mathrm{P} 005543 / 1$, EP/K029843/1, EP/ $\mathrm{K} 010298 / 1, \mathrm{EP} / \mathrm{J017361/1,} \mathrm{EP/M025020/1,} \mathrm{EP/J009016/1,}$ and EP/K016288/1). K.E.J. thanks the Royal Society for a University Research Fellowship and I.D.W.S. acknowledges a Royal Society Wolfson Research Merit Award. B.R. thanks the EPSRC CDT in Theory and Simulation of Materials for a Ph.D. studentship. E.R.J. thanks the Natural Sciences and Engineering Research Council of Canada (NSERC) for funding. The authors thank Dr. Louise Price, UCL, for assistance with the DMACRYS software and Profs. Sally Price and Graeme Day for discussions about the CSP calculations. We acknowledge ARCHER time through the Materials Chemistry Consortium (EP/L000202). CHESS is supported by the NSF and NIH/ NIGMS via NSF award DMR-1332208.

\section{REFERENCES}

(1) Reinitzer, F. Beiträge Zur Kenntniss Des Cholesterins. Monatsh. Chem. 1888, 9, 421-441.

(2) Brandt, J. R.; Salerno, F.; Fuchter, M. J. The Added Value of Small-Molecule Chirality in Technological Applications. Nat. Rev. Chem. 2017, 1, 45.

(3) Göhler, B.; Hamelbeck, V.; Markus, T. Z.; Kettner, M.; Hanne, G. F.; Vager, Z.; Naaman, R.; Zacharias, H. Spin Selectivity in Electron Transmission Through Self-Assembled Monolayers of DoubleStranded DNA. Science 2011, 331, 894-897.

(4) Michaeli, K.; Kantor-Uriel, N.; Naaman, R.; Waldeck, D. H. The Electron's Spin and Molecular Chirality - How Are They Related and How Do They Affect Life Processes? Chem. Soc. Rev. 2016, 45, 64786487.

(5) Manoli, K.; Magliulo, M.; Torsi, L. Chiral Sensor Devices for Differentiation of Enantiomers. Top. Curr. Chem. 2013, 341, 133-176.

(6) Geng, Y.; Trajkovska, A.; Culligan, S. W.; Ou, J. J.; Chen, H. M. P.; Katsis, D.; Chen, S. H. Origin of Strong Chiroptical Activities in Films of Nonafluorenes with a Varying Extent of Pendant Chirality. J. Am. Chem. Soc. 2003, 125, 14032-14038.

(7) Yang, Y.; da Costa, R. C.; Smilgies, D.-M.; Campbell, A. J.; Fuchter, M. J. Induction of Circularly Polarized Electroluminescence from an Achiral Light-Emitting Polymer via a Chiral Small-Molecule Dopant. Adv. Mater. 2013, 25, 2624-2628.

(8) Zinna, F.; Giovanella, U.; Di Bari, L. Highly Circularly Polarized Electroluminescence from a Chiral Europium Complex. Adv. Mater. 2015, 27, 1791-1795.

(9) Li, T.-Y.; Jing, Y.-M.; Liu, X.; Zhao, Y.; Shi, L.; Tang, Z.; Zheng, Y.-X.; Zuo, J.-L. Circularly Polarised Phosphorescent Photoluminescence and Electroluminescence of Iridium Complexes. Sci. Rep. 2015, 5, 14912.

(10) Brandt, J. R.; Wang, X.; Yang, Y.; Campbell, A. J.; Fuchter, M. J. Circularly Polarized Phosphorescent Electroluminescence with a High Dissymmetry Factor from PHOLEDs Based on a Platinahelicene. J. Am. Chem. Soc. 2016, 138, 9743-9746.

(11) Zinna, F.; Pasini, M.; Galeotti, F.; Botta, C.; Di Bari, L.; Giovanella, U. Design of Lanthanide-Based OLEDs with Remarkable Circularly Polarized Electroluminescence. Adv. Funct. Mater. 2017, 27, 1603719

(12) Gilot, J.; Abbel, R.; Lakhwani, G.; Meijer, E. W.; Schenning, A. P. H. J.; Meskers, S. C. J. Polymer Photovoltaic Cells Sensitive to the Circular Polarization of Light. Adv. Mater. 2010, 22, E131-E134.

(13) Korevaar, P. A.; de Greef, T. F. A.; Meijer, E. W. Pathway Complexity in $\pi$-Conjugated Materials. Chem. Mater. 2014, 26, 576586.

(14) Satrijo, A.; Meskers, S. C. J.; Swager, T. M. Probing a Conjugated Polymer's Transfer of Organization-Dependent Properties from Solutions to Films. J. Am. Chem. Soc. 2006, 128, 9030-9031.
(15) Satrijo, A.; Swager, T. M. Facile Control of Chiral Packing in Poly( $p$-Phenylenevinylene) Spin-Cast Films. Macromolecules 2005, 38, 4054-4057.

(16) Yang, Y.; da Costa, R. C.; Fuchter, M. J.; Campbell, A. J. Circularly Polarized Light Detection by a Chiral Organic Semiconductor Transistor. Nat. Photonics 2013, 7, 634-638.

(17) Shen, Y.; Chen, C.-F. Helicenes: Synthesis and Applications. Chem. Rev. 2012, 112, 1463-1535.

(18) Sánchez-Carnerero, E. M.; Agarrabeitia, A. R.; Moreno, F.; Maroto, B. L.; Muller, G.; Ortiz, M. J.; de la Moya, S. Circularly Polarized Luminescence from Simple Organic Molecules. Chem. - Eur. J. 2015, 21, 13488-13500.

(19) Henson, Z. B.; Müllen, K.; Bazan, G. C. Design Strategies for Organic Semiconductors beyond the Molecular Formula. Nat. Chem. 2012, 4, 699-704.

(20) Balandina, T.; van der Meijden, M. W.; Ivasenko, O.; Cornil, D.; Cornil, J.; Lazzaroni, R.; Kellogg, R. M.; De Feyter, S. Self-Assembly of an Asymmetrically Functionalized [6] helicene at Liquid/solid Interfaces. Chem. Commun. 2013, 49, 2207-2209.

(21) Parschau, M.; Fasel, R.; Ernst, K.-H. Coverage and Enantiomeric Excess Dependent Enantiomorphism in Two-Dimensional Molecular Crystals. Cryst. Growth Des. 2008, 8, 1890-1896.

(22) Hauke, C. M.; Rahe, P.; Nimmrich, M.; Schütte, J.; Kittelmann, M.; Stará, I. G.; Starý, I.; Rybáček, J.; Kühnle, A. Molecular SelfAssembly of Enantiopure Heptahelicene-2-Carboxylic Acid on Calcite (1014). J. Phys. Chem. C 2012, 116, 4637-4641.

(23) Ascolani, H.; van der Meijden, M. W.; Cristina, L. J.; Gayone, J. E.; Kellogg, R. M.; Fuhr, J. D.; Lingenfelder, M. Van Der Waals Interactions in the Self-Assembly of 5-amino[6]helicene on $\mathrm{Cu}(100)$ and $\mathrm{Au}(111)$. Chem. Commun. 2014, 50, 13907-13909.

(24) Seibel, J.; Allemann, O.; Siegel, J. S.; Ernst, K.-H. Chiral Conflict among Different Helicenes Suppresses Formation of One Enantiomorph in 2D Crystallization. J. Am. Chem. Soc. 2013, 135, 7434-7437.

(25) Stöhr, M.; Boz, S.; Schär, M.; Nguyen, M.-T.; Pignedoli, C. A.; Passerone, D.; Schweizer, W. B.; Thilgen, C.; Jung, T. A.; Diederich, F. Self-Assembly and Two-Dimensional Spontaneous Resolution of Cyano-Functionalized [7]Helicenes on $\mathrm{Cu}(111)$. Angew. Chem., Int. Ed. 2011, 50, 9982-9986.

(26) Fasel, R.; Parschau, M.; Ernst, K.-H. Amplification of Chirality in Two-Dimensional Enantiomorphous Lattices. Nature 2006, 439, 449452.

(27) Ernst, K.-H. Stereochemical Recognition of Helicenes on Metal Surfaces. Acc. Chem. Res. 2016, 49, 1182-1190.

(28) Hatakeyama, T.; Hashimoto, S.; Oba, T.; Nakamura, M. Azaboradibenzo[6]helicene: Carrier Inversion Induced by Helical Homochirality. J. Am. Chem. Soc. 2012, 134, 19600-19603.

(29) Pop, F.; Auban-Senzier, P.; Frąckowiak, A.; Ptaszyński, K.; Olejniczak, I.; Wallis, J. D.; Canadell, E.; Avarvari, N. Chirality Driven Metallic versus Semiconducting Behavior in a Complete Series of Radical Cation Salts Based on Dimethyl-Ethylenedithio-Tetrathiafulvalene (DM-EDT-TTF). J. Am. Chem. Soc. 2013, 135, 17176-17186.

(30) Pop, F.; Auban-Senzier, P.; Canadell, E.; Rikken, G. L. J. A.; Avarvari, N. Electrical Magnetochiral Anisotropy in a Bulk Chiral Molecular Conductor. Nat. Commun. 2014, 5, 3757.

(31) Zhu, Y.; Gergel, N.; Majumdar, N.; Harriott, L. R.; Bean, J. C.; $\mathrm{Pu}, \mathrm{L}$. First Optically Active Molecular Electronic Wires. Org. Lett. 2006, 8, 355-358.

(32) Josse, P.; Favereau, L.; Shen, C.; Dabos-Seignon, S.; Blanchard, P.; Cabanetos, C.; Crassous, J. Enantiopure versus Racemic Naphthalimide End-Capped Helicenic Non-Fullerene Electron Acceptors: Impact on Organic Photovoltaics Performance. Chem. Eur. J. 2017, 23, 6277-6281.

(33) Shang, X.; Song, I.; Ohtsu, H.; Lee, Y. H.; Zhao, T.; Kojima, T.; Jung, J. H.; Kawano, M.; Oh, J. H. Supramolecular Nanostructures of Chiral Perylene Diimides with Amplified Chirality for High-Performance Chiroptical Sensing. Adv. Mater. 2017, 29, 1605828.

(34) Zerdan, R. B.; Shewmon, N. T.; Zhu, Y.; Mudrick, J. P.; Chesney, K. J.; Xue, J.; Castellano, R. K. The Influence of Solubilizing 
Chain Stereochemistry on Small Molecule Photovoltaics. Adv. Funct. Mater. 2014, 24, 5993-6004.

(35) Sugawara, K.; Nakamura, N.; Yamane, Y.; Hayase, S.; Nokami, T.; Itoh, T. Influence of Chirality on the Cyclohexene-Fused $\mathrm{C}_{60}$ Fullerene Derivatives as an Accepter Partner in a Photovoltaic Cell. Green Energy Environ 2016, 1, 149-155.

(36) Liu, J.; Zhang, Y.; Phan, H.; Sharenko, A.; Moonsin, P.; Walker, B.; Promarak, V.; Nguyen, T.-Q. Effects of Stereoisomerism on the Crystallization Behavior and Optoelectrical Properties of Conjugated Molecules. Adv. Mater. 2013, 25, 3645-3650.

(37) Mîšek, J.; Teplý, F.; Stará, I. G.; Tichý, M.; Šaman, D.; Císařová, I.; Vojtíšek, P.; Starý, I. A Straightforward Route to Helically Chiral NHeteroaromatic Compounds: Practical Synthesis of Racemic 1,14diaza[5]helicene and Optically Pure 1- and 2-aza[6]helicenes. Angew. Chem., Int. Ed. 2008, 47, 3188-3191.

(38) Brandt, J. R.; Pospísil, L.; Bednárová, L.; Correa da Costa, R.; White, A. J. P.; Mori, T.; Teplý, F.; Fuchter, M. J. Intense RedoxDriven Chiroptical Switching with a $580 \mathrm{mV}$ Hysteresis Actuated Through Reversible Dimerization of an Azoniahelicene. Chem. Commun. 2017, in press DOI: 10.1039/C7CC04903J.

(39) Hiszpanski, A. M.; Baur, R. M.; Kim, B.; Tremblay, N. J.; Nuckolls, C.; Woll, A. R.; Loo, Y.-L. Tuning Polymorphism and Orientation in Organic Semiconductor Thin Films via Post-Deposition Processing. J. Am. Chem. Soc. 2014, 136, 15749-15756.

(40) Price, S. L. Predicting Crystal Structures of Organic Compounds. Chem. Soc. Rev. 2014, 43, 2098-2111.

(41) Jones, J. T. A.; Hasell, T.; Wu, X.; Bacsa, J.; Jelfs, K. E.; Schmidtmann, M.; Chong, S. Y.; Adams, D. J.; Trewin, A.; Schiffman, F.; Cora, F.; Slater, B.; Steiner, A.; Day, G. M.; Cooper, A. I. Modular and Predictable Assembly of Porous Organic Molecular Crystals. Nature 2011, 474, 367-371.

(42) Otero-de-la-Roza, A.; Johnson, E. R. A Benchmark for NonCovalent Interactions in Solids. J. Chem. Phys. 2012, 137, 54103.

(43) Otero-de-la-Roza, A.; Cao, B. H.; Price, I. K.; Hein, J. E.; Johnson, E. R. Predicting the Relative Solubilities of Racemic and Enantiopure Crystals by Density-Functional Theory. Angew. Chem., Int. Ed. 2014, 53, 7879-7882.

(44) Bardwell, D. A.; Adjiman, C. S.; Arnautova, Y. A.; Bartashevich, E.; Boerrigter, S. X. M.; Braun, D. E.; Cruz-Cabeza, A. J.; Day, G. M.; Della Valle, R. G.; Desiraju, G. R.; van Eijck, B. P.; Facelli, J. C.; Ferraro, M. B.; Grillo, D.; Habgood, M.; Hofmann, D. W. M.; Hofmann, F.; Jose, K. V. J.; Karamertzanis, P. G.; Kazantsev, A. V.; et al. Towards Crystal Structure Prediction of Complex Organic Compounds - a Report on the Fifth Blind Test. Acta Crystallogr., Sect. B: Struct. Sci. 2011, B67, 535-551.

(45) Whittleton, S. R.; Otero-de-la-Roza, A.; Johnson, E. R. Exchange-Hole Dipole Dispersion Model for Accurate Energy Ranking in Molecular Crystal Structure Prediction. J. Chem. Theory Comput. 2017, 13, 441-450.

(46) Nelson, J.; Kwiatkowski, J. J.; Kirkpatrick, J.; Frost, J. M. Modeling Charge Transport in Organic Photovoltaic Materials. Acc. Chem. Res. 2009, 42, 1768-1778.

(47) Xiao, C.; Kan, X.; Liu, C.; Jiang, W.; Zhao, G.; Zhao, Q.; Zhang, L.; Hu, W.; Wang, Z.; Jiang, L. Controlled Formation of Large-Area Single-Crystalline TIPS-Pentacene Arrays through Superhydrophobic Micropillar Flow-Coating. J. Mater. Chem. C 2017, 5, 2702-2707.

(48) Akkerman, H. B.; Li, H.; Bao, Z. TIPS-Pentacene Crystalline Thin Film Growth. Org. Electron. 2012, 13, 2056-2062.

(49) Vaklev, N. L.; Müller, R.; Muir, B. V. O.; James, D. T.; Pretot, R.; van der Schaaf, P.; Genoe, J.; Kim, J.-S.; Steinke, J. H. G.; Campbell, A. J. High-Performance Flexible Bottom-Gate Organic Field-Effect Transistors with Gravure Printed Thin Organic Dielectric. Adv. Mater. Interfaces 2014, 1, 1300123.

(50) Weimar, M.; Correa da Costa, R.; Lee, F.-H.; Fuchter, M. J. A Scalable and Expedient Route to 1-Aza[6] helicene Derivatives and Its Subsequent Application to a Chiral-Relay Asymmetric Strategy. Org. Lett. 2013, 15, 1706-1709.

(51) Frisch, M. J.; Trucks, G. W.; Schlegel, H. B.; Scuseria, G. E.; Robb, M. A.; Cheeseman, J. R.; Scalmani, G.; Barone, V.; Petersson, G.
A.; Nakatsuji, H.; Li, X.; Caricato, M.; Marenich, A. V.; Bloino, J.; Janesko, B. G.; Gomperts, R.; Mennucci, B.; Hratchian, H. P.; Ortiz, J. V.; Izmaylov, A. F.; Sonnenberg, J. L.; Williams-Young, D.; Ding, F.; Lipparini, F.; Egidi, F.; Goings, J.; Peng, B.; Petrone, A.; Henderson, T.; Ranasinghe, D.; Zakrzewski, V. G.; Gao, J.; Rega, N.; Zheng, G.; Liang, W.; Hada, M.; Ehara, M.; Toyota, K.; Fukuda, R.; Hasegawa, J.; Ishida, M.; Nakajima, T.; Honda, Y.; Kitao, O.; Nakai, H.; Vreven, T.; Throssell, K.; Montgomery, J. A., Jr.; Peralta, J. E.; Ogliaro, F.; Bearpark, M. J.; Heyd, J. J.; Brothers, E. N.; Kudin, K. N.; Staroverov, V. N.; Keith, T. A.; Kobayashi, R.; Normand, J.; Raghavachari, K.; Rendell, A. P.; Burant, J. C.; Iyengar, S. S.; Tomasi, J.; Cossi, M.; Millam, J. M.; Klene, M.; Adamo, C.; Cammi, R.; Ochterski, J. W.; Martin, R. L.; Morokuma, K.; Farkas, O.; Foresman, J. B.; Fox, D. J. Gaussian 09, Revision 1A; Gaussian, Inc.: Wallingford, CT2016.

(52) Karamertzanis, P. G.; Pantelides, C. C. Ab Initio Crystal Structure Prediction-I. Rigid Molecules. J. Comput. Chem. 2005, 26, 304-324.

(53) Karamertzanis, P. G.; Pantelides, C. C. Ab Initio Crystal Structure Prediction. II. Flexible Molecules. Mol. Phys. 2007, 105, 273-291.

(54) Price, S. L.; Leslie, M.; Welch, G. W. A.; Habgood, M.; Price, L. S.; Karamertzanis, P. G.; Day, G. M. Modelling Organic Crystal Structures Using Distributed Multipole and Polarizability-Based Model Intermolecular Potentials. Phys. Chem. Chem. Phys. 2010, 12, 84788490.

(55) Williams, D. E. Improved Intermolecular Force Field for Molecules Containing H, C, N, and O Atoms, with Application to Nucleoside and Peptide Crystals. J. Comput. Chem. 2001, 22, 11541166.

(56) Stone, A. J. Distributed Multipole Analysis, or How to Describe a Molecular Charge Distribution. Chem. Phys. Lett. 1981, 83, 233-239.

(57) Stone, A. J. Distributed Multipole Analysis of Gaussian Wavefunctions, GDMA, Version 2.2.02; University of Cambridge: Cambridge, UK, 2005.

(58) Becke, A. D. On the Large-gradient Behavior of the Density Functional Exchange Energy. J. Chem. Phys. 1986, 85, 7184-7187.

(59) Perdew, J. P.; Burke, K.; Ernzerhof, M. Generalized Gradient Approximation Made Simple. Phys. Rev. Lett. 1996, 77, 3865-3868.

(60) Becke, A. D.; Johnson, E. R. Exchange-Hole Dipole Moment and the Dispersion Interaction Revisited. J. Chem. Phys. 2007, 127, 154108 .

(61) Otero-de-la-Roza, A.; Johnson, E. R. Van Der Waals Interactions in Solids Using the Exchange-Hole Dipole Moment Model. J. Chem. Phys. 2012, 136, 174109.

(62) Blöchl, P. E. Projector Augmented-Wave Method. Phys. Rev. B: Condens. Matter Mater. Phys. 1994, 50, 17953-17979.

(63) Giannozzi, P.; Baroni, S.; Bonini, N.; Calandra, M.; Car, R.; Cavazzoni, C.; Ceresoli, D.; Chiarotti, G. L.; Cococcioni, M.; Dabo, I.; Dal Corso, A.; de Gironcoli, S.; Fabris, S.; Fratesi, G.; Gebauer, R.; Gerstmann, U.; Gougoussis, C.; Kokalj, A.; Lazzeri, M.; Martin-Samos, L.; et al. QUANTUM ESPRESSO: A Modular and Open-Source Software Project for Quantum Simulations of Materials. J. Phys.: Condens. Matter 2009, 21, 395502.

(64) Johnson, E. R. Erin Johnson's group wiki. http://schooner.chem. dal.ca/wiki/XDM (accessed March 17, 2017).

(65) Kirkpatrick, J. An Approximate Method for Calculating Transfer Integrals Based on the ZINDO Hamiltonian. Int. J. Quantum Chem. 2008, 108, 51-56.

(66) Nelsen, S. F.; Blackstock, S. C.; Kim, Y. Estimation of Inner Shell Marcus Terms for Amino Nitrogen Compounds by Molecular Orbital Calculations. J. Am. Chem. Soc. 1987, 109, 677-682.

(67) Stehr, V.; Pfister, J.; Fink, R. F.; Engels, B.; Deibel, C. FirstPrinciples Calculations of Anisotropic Charge-Carrier Mobilities in Organic Semiconductor Crystals. Phys. Rev. B: Condens. Matter Mater. Phys. 2011, 83, 155208. 